\title{
Microstructural characterization of ferroelectric oxides thin fillms based on hafnia
}

\author{
C. Radu ${ }^{1, *}$, C. M. Istrate ${ }^{1}$, C. Ghica ${ }^{1}$ \\ 1 National Institute of Materials Physics, 77125 Măgurele, Romania
}

* Correspondence: cristian.radu@infim.ro

Since the discovery of ferroelectricity in $\mathrm{HfO}_{2}$ thin films, $\mathrm{HfO}_{2}$ based materials have become of great interest for applications such as non-volatile random-access memory devices. Morphological and atomic scale structural investigations of nanomaterials are important in further understanding of their electrical properties. The electrical behavior $(\mathrm{C}-\mathrm{V}, \mathrm{I}-\mathrm{V}$ characteristics and polarization hysteresis loop) of multilayer structures is greatly influenced by the quality of the deposited thin films and also the quality of the interfaces between the layers.

Transmission Electron Microscopy (TEM) is the most appropriate microstructural characterization technique able to provide morphological and structural information about the thin films and their interfaces. We study the morphological properties of this oxide based on hafnia because the orthorhombic phase exhibits ferroelectric properties.

In this work we use a Cs probe-corrected JEM ARM 200F electron microscope, TEMSAED and HRTEM techniques to investigate the morphological structure of $\mathrm{HfO}_{2}$ based thin films and to identify the crystalline phases of the ferroelectric oxide. The studied samples consist in thin films of $\mathrm{HfO}_{2}$ deposited on a TiN electrode and the latter was deposited on a $\mathrm{Si}$ (100) substrate with a native $\mathrm{SiO}_{2}$ layer.

The thin films were grown by atomic layer deposition (ALD) using TEMAHf and ZyALD precursors deposited at $300^{\circ} \mathrm{C}$. HRTEM technique combined with FFT (Fast Fourier Transform) analysis provides a complete characterization of morphological and structural properties of the thin film.

Keywords: ferroelectric oxides, TEM, atomic layer deposition.

\section{Funding}

Not applicable.

\section{Acknowledgments}

Not applicable.

\section{Conflicts of Interest}

The authors declare no conflict of interest. 


\section{Radu, C. M. Istrate, C. Ghica}
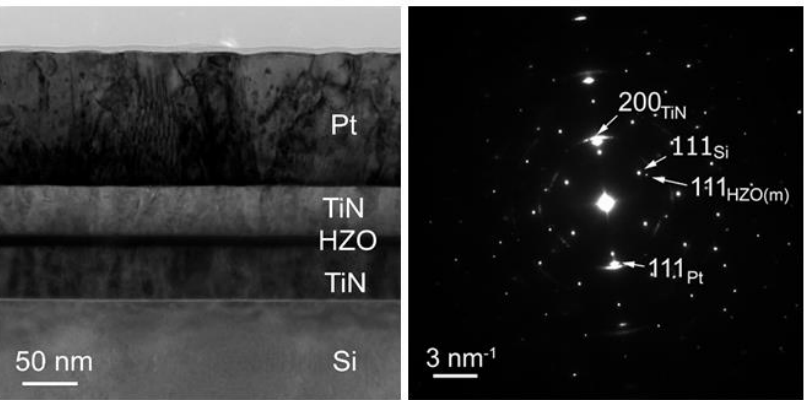

Figure 1. TEM image and corresponding SAED pattern of the Pt/TiN/HZO/TiN/Si heterostructure showing multiple crystalline phases.
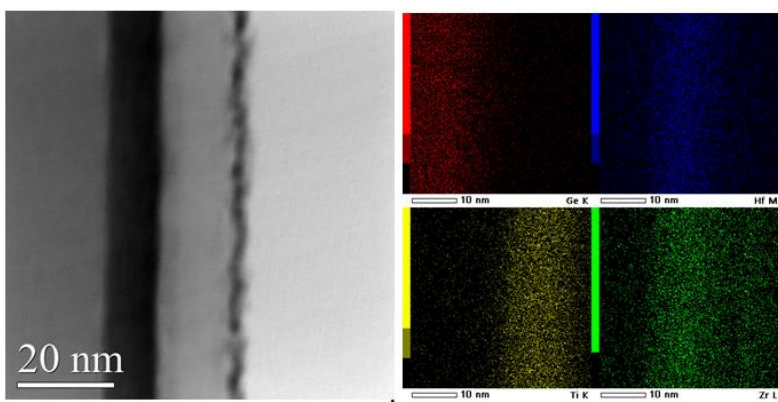

Figure 2. STEM image of TiN/HZO/Ge heterostructure and STEM- EDS map of Ti, Hf, Zr, Ge showing the elemental spatial distribution.

\section{References}

1. Hoffmann, M.; Fengler, F.P.G.; Herzig, M.; Mittmann, T.; Max, B.; Schroeder, U.; Negrea, R.; Pintilie, L.; Slesazeck, S.; Mikolajick, T. Unveiling the double-well energy landscape in a ferroelectric layer. Nature 2019, 565, 464-467, https://doi.org/10.1038/s41586-018-0854-z.
2. Everett, D.; Grimley, T.S.; Mikolajick, T.; Schroeder, Y.; LeBeau, J.M. Atomic Structure of Domain and Interphase Boundaries in Ferroelectric HfO2. Advanced Materials Interfaces 2018, 5, 1701258, https://doi.org/10.1002/admi.201701258.

(C) 2019 by the authors. This article is an open access article distributed under the terms and conditions of the Creative Commons Attribution (CC BY) license (http://creativecommons.org/licenses/by/4.0/). 